\title{
Further Report on Relationship between Blood Platelet Count of Lactating Mothers with Different Arakawa's Reaction and Treatment with Vitamin B.*
}

r33rd Report of the Peroxidase Reaction.

By

Shizuo Kimura.

(木 村箐雄)

(From the Department of Pediatrics, Faculty of Medicine, Tohoku Imperial University, Sendai.

Director: Prof. A. Sato.)

\section{Introduction.}

In the two preceding papers ${ }^{1 / 2)}$ I stated that mothers secreting milk negative to Arakawa's reaction showed, as was formerly reported by J. Kimura, ${ }^{3)^{45}}$ an increase of blood platelet count, and that mother's taking vitamin $B$ showed a less remarkable increase of the count than those with no vitamin intake. An increase of blood platelet count in beriberi cases has already been reported by $\mathrm{Id}{ }_{0}{ }^{6}$ ) $\mathrm{Kubo},{ }^{7} \mathrm{Nakamura}{ }^{8}$ and Iijima. ${ }^{92}$ That a high platelet count is a sign of avitaminosis $\mathrm{B}$ is evident from my own results ${ }^{1-2}$ too.

Now, J. Kimura ${ }^{5}$ administered vitamin B to those mothers with weak or negative Arakawa's reaction and saw a decrease of the platelet count in 17 cases. I desire to report in the present paper of a

* Vitamin $\mathrm{B}_{1}$, unless otherwise noted.

1) Sh. Kim a r a, Tohoku J. Exp. Med., 1939, 37, 241.

2) Sh. Kimura, Tohoku J. Exp. Med., 1940, 38, 210.

3) J. Kim u ra, Tohoku J. Ex. Med., 1934, 23, 494.

4) J. K i m u ra, Tohoku J. Exp. Med., 1985, 27, 360.

5) J. Ki m u ra, Tokoku J. Exp. Med., 1935, 27, 374.

6) Y. Id o, Fukuoka Ikwadaigaku Zassi, 1912, 6, 217.

7) T. K u bo, Tokyo Iji Sinsi, 1921, 1417 .

8) M. N a ka mu ra, Nippon Naikwagakkwai Zassi, 1995, 13, 317.

9) R. Iijim a, Rinsho Byori Ketsuekigakkwai Zassi, 1935, 4, $53 \mathrm{~L}$. 
similar clinical experiment in a much larger number of materials-62 cases in all-with 11 control cases.

\section{Method of Experiment.}

1. Materials. Blood was taken from healthy lactating mothers of breast-fed patients who visited our Dispensary. Blood taking took place twice or more than twice in each case. There were a few mothers who were taking a vitamin $\mathrm{B}$ preparation before visiting, although they were not suffering from beriberi.

2. A rakawa's reaction. Milk was examined with Arakawa's reagent in each case.

3. Platelet count. I used $\mathrm{L}$ a m pert' $\mathrm{s}^{10}$ thrombocyte counting method. The ear lobe was cleaned with ether, and a cut was made with Francke's needle. Blood was taken into the "Athrombit" and diluted with 'Tyrode's solution. After shaking the pipette for a few minutes, the sixth drop of the contents was examined for blood platelets, waiting over 5 minutes after counting chamber was filled. Blood taking took place between 11 and 12 o'clock a.m., when mothers were in a fasting state.

4. Vitamin B preparations. Vitamin B preparations were used perorally or were injected subcutaneously as vitamin $B$ complex or $B_{1}$.

\section{Result of Experiment.}

I examined a change of blood platelet count and A rakawa's reaction in 62 cases of lactating mothers treated with vitamin $B$. Blood taking was performed 164 times. For control experiment I examined the change of blood platelet count and A raka wa's reaction in 11 cases of lactating mothers not subject to vitamin B treatment.

The results were as follows.

1. As will be seen from Table 1, 29 mother cases showed a parallelism between a decrease of the platelet count and an improvement of A rakawa's reaction, on an administration of vitamin B. In Cases Nos. $1,3,11,13,19,20,21,22,24,25,28$ and 29 the detoxicating hormone of the liver, yakriton, was used for a better mobilization of vitamin B (Cf. Takamats u's'1) paper). In Cases Nos. 1, 2, 3, 4, 5, 6, 8, 17, 22 and 24 Arakaw a's reaction improved and changed from nega-

10) H. L ampert, Verh. Dtsch. Ges. inn. Med, 1931, 43, 92.

11) A. Takam ats u, Tohoku J. Exp. Med, 1934, 23, 372. 
TAble

Group of lactating mothers, in whom a parallelism between a decrease of blood

\begin{tabular}{|c|c|c|c|c|c|}
\hline \multirow{2}{*}{$\begin{array}{c}\text { Case } \\
\text { No. and } \\
\text { name }\end{array}$} & \multirow{2}{*}{$\begin{array}{l}\text { Age of } \\
\text { mother } \\
\text { (vears) } \\
\text { and age } \\
\text { of infant } \\
\text { (months) }\end{array}$} & \multirow{2}{*}{$\begin{array}{c}\text { Day of } \\
\text { experi- } \\
\text { ment }\end{array}$} & \multirow{2}{*}{$\begin{array}{l}\text { Blood } \\
\text { platelet } \\
\text { count } \\
\text { (thou- } \\
\text { sands) } \\
\text { per emm. }\end{array}$} & \multicolumn{2}{|c|}{ Arakawa's reaction } \\
\hline & & & & $\underset{\left(0^{\prime}\right)\left(1^{\prime}\right)\left(2^{\prime}\right)\left(3^{\prime}\right)\left(4^{\prime}\right)\left(5^{\prime}\right)}{\text { Right }}$ & $\begin{array}{c}\text { Left } \\
\left(0^{\prime}\right)\left(1^{\prime}\right)\left(2^{\prime}\right)\left(3^{\prime}\right)\left(4^{\prime}\right)\left(5^{\prime}\right)\end{array}$ \\
\hline C. $\stackrel{1}{T}$ & $\begin{array}{r}32 \\
2\end{array}$ & $\begin{array}{l}\text { 15. IX } \\
20 . ", \\
25 . " 3 " \\
16 . X\end{array}$ & $\begin{array}{l}510 \\
460 \\
360 \\
480\end{array}$ & $\begin{array}{l}-+H \text { H H H } \\
+H \text { H.H H H } \\
\pm H \text { H H H H } \\
\text { H H H H H }\end{array}$ & $\begin{array}{lllll}+ & H & H & H \\
+ & H & H & H & H \\
- & H & H & H \\
- & H & H & H\end{array}$ \\
\hline H. $\mathrm{2}$. & $\begin{array}{r}35 \\
9\end{array}$ & $\begin{array}{l}\text { 16. III } \\
27 . "\end{array}$ & $\begin{array}{l}430 \\
2=0\end{array}$ & $\begin{array}{l} \pm++H+H \\
+H H H\end{array}$ & $\begin{array}{l} \pm++H+H \\
+H+H+H \text { H }\end{array}$ \\
\hline S. $\stackrel{3}{H}$ & $\begin{array}{r}31 \\
4\end{array}$ & $\begin{array}{l}\text { 18. VIII } \\
23 . ",\end{array}$ & $\begin{array}{l}460 \\
320\end{array}$ & $\begin{array}{l} \pm+H \text { H } H \text { H } \\
-\# \text { H H H H }\end{array}$ & $\begin{array}{l} \pm \pm+十 \frac{H}{H}+\frac{H}{H} \\
+H H \text { H H }\end{array}$ \\
\hline S. $\mathrm{A}$. & $\begin{array}{l}38 \\
13\end{array}$ & $\begin{array}{l}23 . \mathrm{X} \\
30 ., "\end{array}$ & $\begin{array}{l}570 \\
330\end{array}$ & $\begin{array}{l} \pm++H+H \\
\pm+H+H+H\end{array}$ & $\begin{array}{l}- \pm \pm \pm++ \\
\pm+H+H+\end{array}$ \\
\hline K. $\stackrel{5}{Y}$. & $\begin{array}{l}22 \\
14\end{array}$ & $\begin{array}{l}\text { 9. VIII } \\
20 . \quad "\end{array}$ & $\begin{array}{l}380 \\
290\end{array}$ & $\begin{array}{l}-+H \mathrm{H} H \mathrm{H} \\
-\mathrm{H} H \mathrm{H}\end{array}$ & $\begin{array}{l}- \pm+++H \\
-H+H+H\end{array}$ \\
\hline K. $\stackrel{6}{\mathrm{C}}$ & $\begin{array}{r}26 \\
3\end{array}$ & $\begin{array}{l}\text { 10. XII } \\
20 . ", \\
30 . ",\end{array}$ & $\begin{array}{l}470 \\
410 \\
350\end{array}$ & 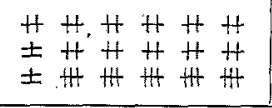 & $\begin{array}{l}- \pm++++ \\
-++++t \\
\pm+4+4+4+4\end{array}$ \\
\hline R. F. & $\begin{array}{l}34 \\
19\end{array}$ & $\begin{array}{l}\text { 29. IX } \\
\text { 5. X }\end{array}$ & $\begin{array}{l}570 \\
420\end{array}$ & $\begin{array}{l}- \pm++t \\
\pm+H+H\end{array}$ & $\begin{array}{l}\text { 二 } \pm++t \\
\pm+H+H+H\end{array}$ \\
\hline Y.S. & $\begin{array}{l}32 \\
16\end{array}$ & $\begin{array}{l}\text { 30. V } \\
\text { 5. VI } \\
11.4\end{array}$ & $\begin{array}{l}390 \\
400 \\
280\end{array}$ & $\begin{array}{l}- \pm+H H+H \\
- \pm \pm \pm+ \\
+H H+H\end{array}$ & $\begin{array}{l}-m \pm \pm \pm \pm \\
-\overline{-}- \pm \frac{-}{H} \\
++H\end{array}$ \\
\hline$\stackrel{9}{\mathbf{g}}$ & $\begin{array}{l}36 \\
10\end{array}$ & $\begin{array}{l}\text { 5. VI } \\
\text { 19. } "\end{array}$ & $\begin{array}{l}480 \\
420\end{array}$ & $\begin{array}{l}- \pm++4+4 \\
-++H+H\end{array}$ & $- \pm \pm \pm++t+4$ \\
\hline $\begin{array}{c}10 \\
\text { K. S. }\end{array}$ & $\begin{array}{r}35 \\
2 \\
5\end{array}$ & $\begin{array}{l}\text { 19. IX } \\
\text { 15. I }\end{array}$ & $\begin{array}{l}480 \\
400\end{array}$ & $-\overline{-} \overline{-}- \pm+$ & $\begin{array}{l} \pm+t+H \\
\pm+t+H\end{array}$ \\
\hline $\begin{array}{l}11 \\
\text { N.I. }\end{array}$ & $\begin{array}{r}23 \\
9\end{array}$ & $\begin{array}{l}\text { 7. I } \\
17 . " . \\
21 . "\end{array}$ & $\begin{array}{l}520 \\
490 \\
400\end{array}$ & $\begin{array}{l}- \pm+++ \\
- \pm++4+4 \\
- \pm+++4\end{array}$ & $\begin{array}{l}- \pm++H \\
-- \pm+H+H \\
-+H+H H\end{array}$ \\
\hline I. $\mathrm{M}$ & $\begin{array}{r}28 \\
3\end{array}$ & $\begin{array}{l}\text { 12. II } \\
\text { 16. " }\end{array}$ & $\begin{array}{l}540 \\
320\end{array}$ & $\begin{array}{l}-\overline{-}- \pm+ \\
- \pm+H\end{array}$ & $\begin{array}{l}--\overline{ \pm}+ \\
- \pm++4\end{array}$ \\
\hline $\begin{array}{c}13 \\
Y . I .\end{array}$ & $\begin{array}{r}25 \\
3\end{array}$ & $\begin{array}{l}\text { 13. II } \\
22 . ",\end{array}$ & $\begin{array}{l}410 \\
320\end{array}$ & $\overline{ \pm} \bar{H} \overline{+} \bar{H} \bar{H}$ & $-\overline{-}+ \pm \pm \pm+4$ \\
\hline $\begin{array}{l}\mathrm{I} 4 \\
\mathrm{~K} . \mathrm{I}\end{array}$ & $\begin{array}{l}31 \\
13\end{array}$ & $\begin{array}{l}\text { 13. VI } \\
18, " s \\
25 .\end{array}$ & $\begin{array}{l}500 \\
350 \\
360\end{array}$ & $\begin{array}{l}-\overline{ \pm} \pm+ \\
- \pm+H+H \\
- \pm+H+H\end{array}$ & $\begin{array}{l}--\overline{-}-\overline{+} \\
-\overline{+}+\mathrm{H}+\mathrm{H}\end{array}$ \\
\hline
\end{tabular}


1.

platelet count and an improvement of A rak awa's reaction was seen.

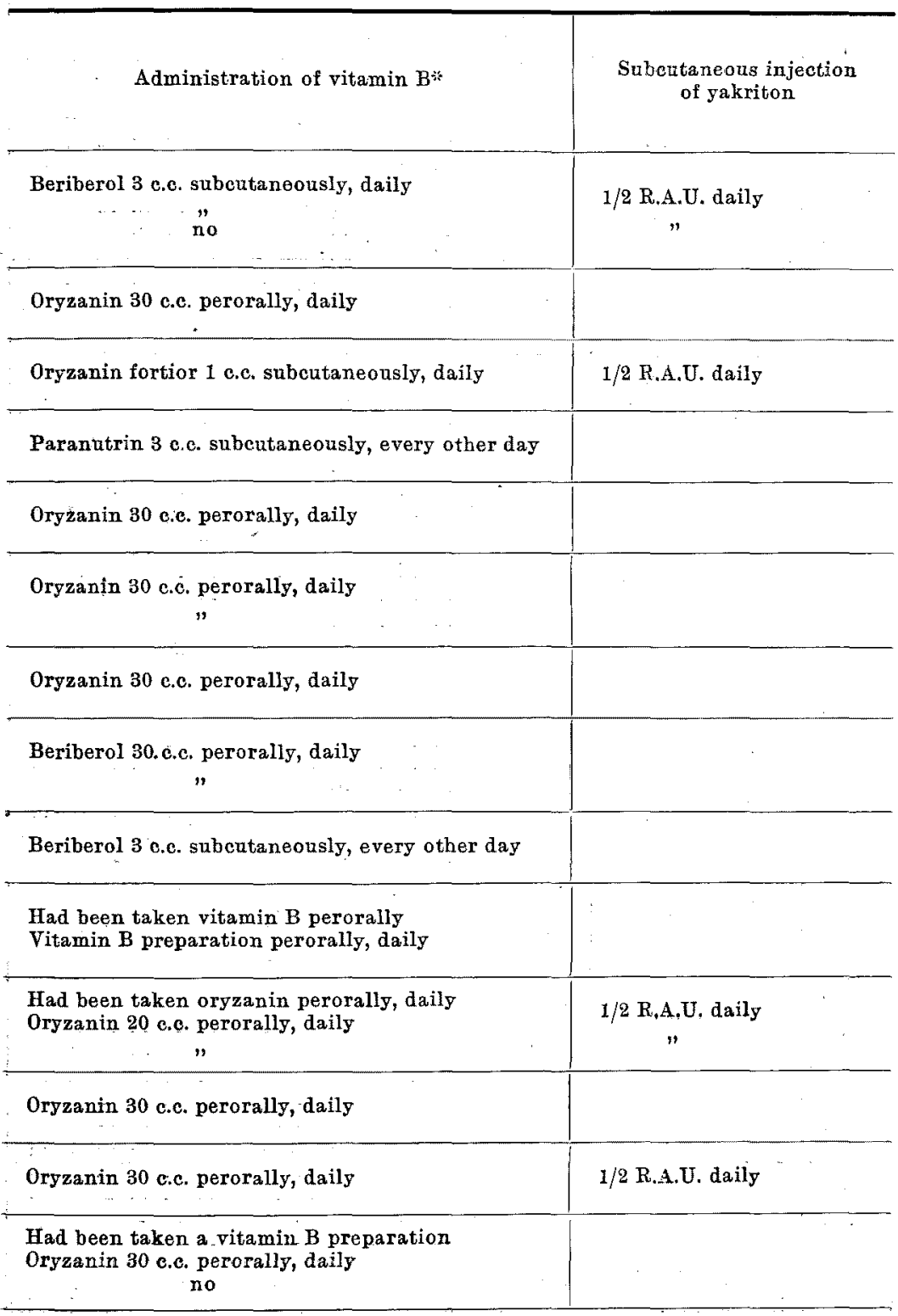


Table 1. (Continued).

\begin{tabular}{|c|c|c|c|c|c|}
\hline \multirow{2}{*}{$\begin{array}{c}\text { Case } \\
\text { No. and } \\
\text { name }\end{array}$} & \multirow{2}{*}{$\begin{array}{l}\text { Age of } \\
\text { mother } \\
\text { (years) } \\
\text { and age } \\
\text { of infant } \\
\text { (months) }\end{array}$} & \multirow{2}{*}{$\begin{array}{l}\text { Day of } \\
\text { experi- } \\
\text { ment }\end{array}$} & \multirow{2}{*}{$\begin{array}{l}\text { Blood } \\
\text { platelet } \\
\text { count } \\
\text { (thou- } \\
\text { sands) } \\
\text { per cmm. }\end{array}$} & \multicolumn{2}{|c|}{ Arakawa's reaction } \\
\hline & & & & $\underset{\left(0^{\prime}\right)\left(1^{\prime}\right)\left(2^{\prime}\right)\left(3^{\prime}\right)\left(4^{\prime}\right)\left(5^{\prime}\right)}{\stackrel{1}{\text { Right }}}$ & $\begin{array}{c}\text { Left } \\
\left(0^{\prime}\right)\left(1^{\prime}\right)\left(2^{\prime}\right)\left(3^{\prime}\right)\left(4^{\prime}\right)\left(5^{\prime}\right)\end{array}$ \\
\hline $\begin{array}{l}15 \\
\mathrm{~T} . \mathrm{K}\end{array}$ & $\begin{array}{r}29 \\
2\end{array}$ & $\begin{array}{l}\text { 15. XI } \\
26 . .3 " \\
\text { 7. XII }\end{array}$ & $\begin{array}{l}480 \\
410 \\
310\end{array}$ & $\begin{array}{l}- \pm \pm \pm \pm \pm \\
- \pm \pm+ \pm H \\
\pm H+H+H\end{array}$ & $\begin{array}{l}-\overline{ \pm} \pm \overline{ \pm} \\
- \pm \pm \\
- \pm H+H\end{array}$ \\
\hline $\begin{array}{l}16 \\
\text { H. } K .\end{array}$ & $\begin{array}{l}38 \\
10\end{array}$ & $\begin{array}{l}\text { 23. XII } \\
\text { I3. I }\end{array}$ & $\begin{array}{l}480 \\
300\end{array}$ & $\begin{array}{l}- \pm \pm \pm \pm \pm \pm \\
- \pm \pm+1\end{array}$ & $- \pm \pm \pm \pm \overline{+}$ \\
\hline $\begin{array}{l}17 \\
\text { T. S. }\end{array}$ & $\begin{array}{l}27 \\
10\end{array}$ & $\begin{array}{r}\text { 3. X } \\
\text { 8. } " \\
12 . "\end{array}$ & $\begin{array}{l}670 \\
530 \\
370\end{array}$ & 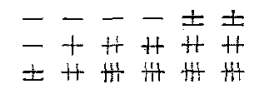 & 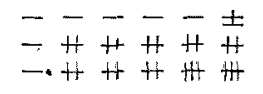 \\
\hline $\begin{array}{l}18 \\
\text { N. I. }\end{array}$ & $\begin{array}{r}32 \\
2\end{array}$ & $\begin{array}{l}\text { 20. X } \\
\text { 25. } " \ddot{X I} \\
\text { 5. XI }\end{array}$ & $\begin{array}{l}860 \\
530 \\
390\end{array}$ & $\begin{array}{l}--\overline{-}-\overline{-} \\
-\bar{t} \\
- \pm+H\end{array}$ & $\begin{array}{l}-- \pm \pm \pm+ \\
-- \pm \pm \pm \pm \\
-+H+H\end{array}$ \\
\hline $\begin{array}{l}19 \\
\text { T. A. }\end{array}$ & $\begin{array}{r}28 \\
8\end{array}$ & $\begin{array}{l}\text { 17. I } \\
24 . .2 \\
\text { I. II }\end{array}$ & $\begin{array}{l}640 \\
620 \\
400\end{array}$ & $\begin{array}{l}-- \pm \pm \pm \pm \\
-\bar{t}+ \pm+1 \\
- \pm \pm+1\end{array}$ & $\begin{array}{l}-\overline{-}-\overline{-} \\
-\overline{ \pm}+ \\
- \pm+H+\end{array}$ \\
\hline $\begin{array}{l}20 \\
\text { F. S. }\end{array}$ & $\begin{array}{l}25 \\
1.5\end{array}$ & $\begin{array}{l}\text { 12. II } \\
17 . " \\
25 . "\end{array}$ & $\begin{array}{l}890 \\
710 \\
440\end{array}$ & $\begin{array}{l}-\overline{-} \pm \pm \\
- \pm \pm \pm \pm \pm \\
-+ \pm+H\end{array}$ & $\begin{array}{l}-\overline{-} \overline{-} \overline{-} \\
-\overline{ \pm} \pm \pm \pm \\
-+ \pm \mathrm{H}\end{array}$ \\
\hline $\begin{array}{l}21 \\
\mathrm{Y} . \mathrm{N}\end{array}$ & $\begin{array}{l}27 \\
13\end{array}$ & $\begin{array}{l}\text { 28. I } \\
\text { 3. II }\end{array}$ & $\begin{array}{l}430 \\
340 \\
\end{array}$ & $\overline{ \pm}+ \pm-4 \pm \frac{ \pm}{H}$ & $\begin{array}{l}-\overline{-} \overline{-} \overline{ \pm} \\
- \pm \pm \pm\end{array}$ \\
\hline$\stackrel{22}{M . K .}$ & $\begin{array}{l}32 \\
10\end{array}$ & $\begin{array}{l}\text { 10. VI } \\
16 . ", \\
25 . "\end{array}$ & $\begin{array}{l}480 \\
360 \\
280 \\
\end{array}$ & & 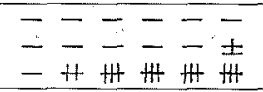 \\
\hline $\begin{array}{l}23 \\
\text { A. I. }\end{array}$ & $\begin{array}{r}34 \\
9\end{array}$ & $\begin{array}{r}\text { 3. VI } \\
11 ., " \\
17 ., n\end{array}$ & $\begin{array}{l}560 \\
490 \\
330 \\
\end{array}$ & $\begin{array}{l}=- \pm- \pm- \\
- \pm \pm \pm \pm+\end{array}$ & $=---\overline{ \pm}-\frac{ \pm}{+H}$ \\
\hline$\stackrel{24}{\text { M. M. }}$ & $\begin{array}{r}25 \\
4\end{array}$ & $\begin{array}{l}\text { I2. VII } \\
\text { 17. " "III } \\
\text { 8. VII }\end{array}$ & $\begin{array}{l}430 \\
300 \\
280 \\
\end{array}$ & $\begin{array}{l}-\overline{-}-\overline{-} \\
-+H+H \\
-H \text { H H } \\
=H\end{array}$ & $\begin{array}{l}-\overline{-}-\overline{-}-\bar{H} \\
-++H+H . H \\
-+H+H \text { H }\end{array}$ \\
\hline $\begin{array}{l}25 \\
\text { Y.S. }\end{array}$ & $\begin{array}{l}39 \\
15\end{array}$ & \begin{tabular}{cc|}
23. & $\mathrm{XI}$ \\
29. & $n$ \\
6. &,
\end{tabular} & $\begin{array}{l}630 \\
680 \\
480 \\
\end{array}$ & $\begin{array}{l}\overline{-}-\overline{-}-\overline{-} \\
\overline{-} \overline{+}+\bar{H}+4\end{array}$ & $\begin{array}{l}-\overline{-}-\overline{-} \\
-\overline{-} \overline{-} \\
- \pm+H\end{array}$ \\
\hline $\begin{array}{l}26 \\
\text { M. K. }\end{array}$ & $\begin{array}{r}26 \\
9\end{array}$ & $\begin{array}{c}\text { 22. XI } \\
2 . \mathrm{XII} \\
11 ., 19 \\
\end{array}$ & $\begin{array}{l}590 \\
490 \\
520 \\
\end{array}$ & $\begin{array}{l}-\overline{-}-\overline{-}- \\
- \pm \overline{-} \overline{-} \\
\bar{H}\end{array}$ & . \\
\hline $\begin{array}{r}27 \\
\mathrm{~K} .0 . \\
\end{array}$ & $\begin{array}{l}27 \\
13\end{array}$ & $\begin{array}{l}\text { 13. II } \\
17 ., n \\
\end{array}$ & $\begin{array}{l}400 \\
340 \\
\end{array}$ & $\begin{array}{l}-\overline{ \pm} \pm \overline{+}+\overline{+} \\
- \pm \overline{+}\end{array}$ & $\begin{array}{l}-\overline{-} \overline{+}-\overline{+} \\
- \pm+4\end{array}$ \\
\hline $\begin{array}{l}28 \\
\text { K. T. }\end{array}$ & $\begin{array}{l}27 \\
1.5\end{array}$ & $\begin{array}{r}6.1 \\
11 . ", \\
18 . " \\
24 . ", \\
\end{array}$ & $\begin{array}{l}490 \\
430 \\
670 \\
400\end{array}$ & $\begin{array}{l}- \pm- \pm-- \\
-- \pm- \pm- \\
-- \pm \pm \pm\end{array}$ & $\begin{array}{l}---\cdots--1 \\
--1- \pm- \\
--1 \pm\end{array}$ \\
\hline $\begin{array}{l}29 \\
\text { K. T. }\end{array}$ & $\begin{array}{r}26 \\
2\end{array}$ & $\begin{array}{l}\text { 16. VII } \\
23 . " 4 \\
30 . " 1 \\
6 . \text { IX }\end{array}$ & $\begin{array}{l}480 \\
310 \\
280 \\
270\end{array}$ & $\begin{array}{l}-----\overline{-} \\
--- \pm \overline{+} \\
-- \pm \pm \\
-- \pm \pm+\end{array}$ & $\begin{array}{l}----\overline{-} \\
--- \pm \pm \\
-- \pm \pm \\
-- \pm \pm \pm\end{array}$ \\
\hline
\end{tabular}

* International units of vitamin $B$ preparations used are as follows :- " Injectio tion contained in $1 \mathrm{cmm}$. $0.05 \mathrm{mg}$. and $0.5 \mathrm{mg}$. of $B_{2}$ crystals $=333$ international units. 


\begin{tabular}{|c|c|}
\hline Administration of vitamin $B *$ & $\begin{array}{l}\text { Subeutaneous injection } \\
\text { of yakriton }\end{array}$ \\
\hline $\begin{array}{c}\text { Oryzanin } 30 \text { c.c. perorally, daily } \\
, "\end{array}$ & \\
\hline Oryzanin 30 c.c. perorally, daily & \\
\hline $\begin{array}{c}\text { Beriberol } 3 \text { c.c. subcutaneously, daily } \\
"\end{array}$ & \\
\hline $\begin{array}{l}\text { Oryzanin } 20 \text { c.c. perorally, daily } \\
\text { Beriberol } 5 \text { e.e. subeutaneously, daily }\end{array}$ & \\
\hline $\begin{array}{c}\text { Had been taking a vitamin B preparation } \\
\text { Oryzanin } 30 \text { c.c. perorally, daily } \\
\text { "" }\end{array}$ & $\begin{array}{c}\text { 1/2 R.A.U. daily } \\
, "\end{array}$ \\
\hline $\begin{array}{c}\text { Oryzanin } 20 \text { c.c. Beriberol } 20 \text { c.c. perorally, daily } \\
\text { " }\end{array}$ & 1/2 R.A.U. daily \\
\hline Beriberol 20 c.c. Oryzanin 20 c.c. perorally, daily & 1/2 R.A.U. daily \\
\hline $\begin{array}{c}\text { Beriberol } 20 \text { c.e. Oryzanin } 20 \text { c.c. perorally, daily } \\
\text { " }\end{array}$ & 1/2 R.A.U. every other day \\
\hline $\begin{array}{c}\text { Beriberol } 20 \text { c.c. Oryzanin } 20 \text { c.c. perorally, daily } \\
\text { " }\end{array}$ & \\
\hline $\begin{array}{c}\text { Had been taking a vitamin B preparation for } 20 \text { days } \\
\text { Paranutrin } 3 \text { c.e. subcutaneously, daily } \\
\text { no }\end{array}$ & $\begin{array}{l}\text { I/2 R.A.U. daily } \\
\text { 1/2 R.A.U. every other day }\end{array}$ \\
\hline $\begin{array}{l}\text { Paranutrin } 5 \text { c.c. subcutaneously, daily } \\
\text { and Oryzanin } 50 \text { e.c. perorally, daily }\end{array}$ & 1/2 R.A.U. daily \\
\hline $\begin{array}{c}\text { Oryzanin } 30 \text { c.c. perorally, daily } \\
\text { " }\end{array}$ & \\
\hline $\begin{array}{l}\text { Last year had taken a vitamin B preparation } \\
\text { Beriberol } 20 \text { c.c. Paranutrin } 20 \text { c.c. perorally, daily }\end{array}$ & \\
\hline $\begin{array}{l}\text { Beriberol } 2 \text { c.c. subcutaneously, daily } \\
\text { Oryzanin } 2 \text { c.c. subcutaneously, daily } \\
\text { Oryzanin fortior } 1 \text { c.c. subcutaneously, daily }\end{array}$ & $\begin{array}{l}\text { 1/2 R.A.U, daily } \\
\text { ", }\end{array}$ \\
\hline $\begin{array}{c}\text { Beriberol 3 c.e. subcutaneously for times } \\
\text { Beriberol } 3 \text { c.c. subcutaneously, daily } \\
\text { ", }\end{array}$ & $\begin{array}{c}\text { I/2 R.A.U. daily } \\
\text { " }\end{array}$ \\
\hline
\end{tabular}

Oryzanin and "Injectio Oryzanin fortior" of Sankyo Co. are the vitamin B $B_{1}$ preparaLiquor Oryzanin, Beriberol, Abery and Paranutrin ; in 100 c.c. $=600,102,208$ and $150 \mathrm{~L}$ U. 
TABLE

Group of lactating mother, in whom only a

\begin{tabular}{|c|c|c|c|c|c|}
\hline \multirow{2}{*}{$\begin{array}{c}\text { Case } \\
\text { No, and } \\
\text { name }\end{array}$} & \multirow{2}{*}{$\begin{array}{l}\text { Age of } \\
\text { mother } \\
\text { (years) } \\
\text { and age } \\
\text { of infant } \\
\text { (months) }\end{array}$} & \multirow{2}{*}{$\begin{array}{c}\text { Day of } \\
\text { experi- } \\
\text { ment }\end{array}$} & \multirow{2}{*}{$\begin{array}{c}\text { Blood } \\
\text { platelet } \\
\text { count } \\
\text { (thou- } \\
\text { sands) } \\
\text { per crnm. }\end{array}$} & \multicolumn{2}{|c|}{ Arakawa's reaction } \\
\hline & & & & $\frac{\text { Right }}{\left(0^{\prime}\right)\left(1^{\prime}\right)\left(\mathbf{2}^{\prime}\right)\left(3^{\prime}\right)\left(4^{\prime}\right)\left(5^{\prime}\right)}$ & $\frac{\text { Leit }}{\left(0^{\prime}\right)\left(1^{\prime}\right)\left(2^{\prime}\right)\left(3^{\prime}\right)\left(4^{\prime}\right)\left(5^{\prime}\right)}$ \\
\hline $\begin{array}{c}1 \\
\text { T. } F .\end{array}$ & $\begin{array}{l}27 \\
19\end{array}$ & $\begin{array}{l}\text { 17. IX } \\
22 . " n \\
29 . " . " \\
6 . X^{\prime \prime} \\
12 . " 1\end{array}$ & $\begin{array}{l}660 \\
570 \\
530 \\
640 \\
480\end{array}$ & 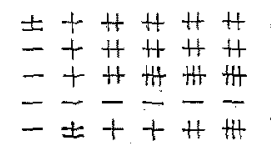 & $\begin{array}{l}- \pm \pm+++ \\
- \pm+++t+ \\
- \pm \pm+ \pm+ \pm \\
- \pm \pm+ \pm\end{array}$ \\
\hline M. T. & $\begin{array}{r}26 \\
9\end{array}$ & $\begin{array}{l}\text { 31. VII } \\
\text { 3. VIII } \\
10 ., ",\end{array}$ & $\begin{array}{l}540 \\
480 \\
340\end{array}$ & $\begin{array}{l}- \pm+++t \\
- \pm \pm+H+H \\
- \pm+H \text { H }\end{array}$ & $\begin{array}{l}- \pm+++4 \\
-\overline{ \pm}+4+4 \\
- \pm H+H\end{array}$ \\
\hline Y. $\stackrel{3}{0}$ & $\begin{array}{l}24 \\
19\end{array}$ & $\begin{array}{l}24 . \mathrm{X} \\
28 . " \Rightarrow\end{array}$ & $\begin{array}{l}610 \\
470\end{array}$ & $\begin{array}{l}-- \pm \pm \pm \\
-- \pm+t+\end{array}$ & $\begin{array}{l} \pm+H H \text { H H } \\
\pm H H+H \text { H }\end{array}$ \\
\hline s. $\stackrel{4}{\text { F. }}$ & $\begin{array}{r}31 \\
4\end{array}$ & $\begin{array}{r}\text { 6. I } \\
11 ., " \\
17 ., "\end{array}$ & $\begin{array}{l}650 \\
560 \\
430\end{array}$ & $\begin{array}{l}- \pm \pm \pm \pm \pm \\
- \pm+H+4 \\
\pm+4+4\end{array}$ & $\begin{array}{l} \pm \pm+4+4 \\
- \pm \pm+4+4\end{array}$ \\
\hline K. $\stackrel{5}{Y}$. & $\begin{array}{r}34 \\
7\end{array}$ & $\begin{array}{l}13.1 \\
20 ., " \\
27 ., "\end{array}$ & $\begin{array}{l}550 \\
450 \\
270\end{array}$ & $\begin{array}{l}- \pm \pm \pm \pm \pm \\
-- \pm \pm \pm \\
--- \pm \pm\end{array}$ & $\begin{array}{l}-H++ \pm+\frac{H}{-}-\bar{H} \\
\overline{ \pm}+\bar{H}\end{array}$ \\
\hline$\stackrel{6}{\mathrm{M}} \cdot \mathrm{M}$. & $\begin{array}{r}25 \\
4\end{array}$ & $\begin{array}{r}29 . X \\
6 . " \\
17 . "\end{array}$ & $\begin{array}{l}720 \\
630 \\
430\end{array}$ & $\begin{array}{l}- \pm \pm+ \pm+ \\
- \pm++H+1 \\
- \pm \pm- \pm \pm\end{array}$ & $\begin{array}{l}- \pm \pm \pm \pm \pm \\
- \pm+ \pm \pm+1 \\
-- \pm \pm\end{array}$ \\
\hline o. $\stackrel{7}{\mathrm{U} .}$ & $\begin{array}{l}30 \\
10\end{array}$ & $\begin{array}{l}\text { 12. II } \\
18 . ", \\
25 . "\end{array}$ & $\begin{array}{l}480 \\
390 \\
300\end{array}$ & $\begin{array}{l}- \pm \pm+1+ \\
-- \pm \pm+1 \\
-- \pm+4+4\end{array}$ & $\begin{array}{l}- \pm \pm++1 \\
-- \pm+1+H\end{array}$ \\
\hline $\begin{array}{c}8 \\
\text { J. } 0 .\end{array}$ & $\begin{array}{r}34 \\
1\end{array}$ & $\begin{array}{l}11 . \mathbf{I I} \\
15 . " n \\
23 . ",\end{array}$ & $\begin{array}{l}540 \\
590 \\
420\end{array}$ & $\begin{array}{l}-- \pm \pm \pm \pm \\
- \pm- \pm \pm+1\end{array}$ & $\begin{array}{l}- \pm \pm+ \pm+ \\
- \pm \pm \pm+ \pm\end{array}$ \\
\hline C. $\stackrel{9}{K}$. & $\begin{array}{r}33 \\
8\end{array}$ & $\begin{array}{l}\text { 28. III } \\
\text { 12. IV } \\
18 ., 9\end{array}$ & $\begin{array}{r}590 \\
470 \\
390\end{array}$ & $\begin{array}{l}- \pm \pm \pm+ \pm \\
-+ \pm+1+1 \\
-- \pm++4\end{array}$ & $\begin{array}{l}-\overline{+} \pm \pm \pm \\
- \pm+ \pm+4 \\
- \pm \pm+ \pm\end{array}$ \\
\hline $\begin{array}{c}10 \\
\mathrm{~K} . \mathrm{M} .\end{array}$ & $\begin{array}{r}33 \\
4\end{array}$ & $\begin{array}{l}\text { 22. } \mathrm{XII} \\
26 . ",\end{array}$ & $\begin{array}{l}520 \\
470\end{array}$ & $- \pm++H+$ & 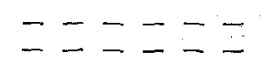 \\
\hline $\begin{array}{c}11 \\
\text { W.S. }\end{array}$ & $\begin{array}{l}29 \\
16\end{array}$ & $\begin{array}{l}\text { 12. VIT } \\
\text { 18. } "\end{array}$ & $\begin{array}{l}530 \\
430\end{array}$ & $\begin{array}{l}--\overline{-}- \pm \\
-\dot{ \pm}\end{array}$ & $\begin{array}{l}- \pm \pm++ \\
- \pm+t+H\end{array}$ \\
\hline $\begin{array}{c}12 \\
M . S .\end{array}$ & $\begin{array}{r}27 \\
9\end{array}$ & $\begin{array}{r}9 . I \\
17 ., " \\
23 ., 4\end{array}$ & $\begin{array}{l}800 \\
570 \\
440\end{array}$ & $\begin{array}{l}---- \pm-5 \\
-- \pm \pm \pm \pm\end{array}$ & $\begin{array}{l}-- \pm \pm \pm \pm \\
-- \pm \pm \pm \pm\end{array}$ \\
\hline $\begin{array}{c}13 \\
\text { K. M. }\end{array}$ & $\begin{array}{l}35 \\
15\end{array}$ & $\begin{array}{l}\text { 4. II } \\
\text { 12. } "\end{array}$ & $\begin{array}{l}430 \\
260\end{array}$ & $\begin{array}{l}-- \pm \pm \pm \\
-- \pm \pm\end{array}$ & $---\div-1$ \\
\hline
\end{tabular}


2.

restoration of blood platelet count was seen.

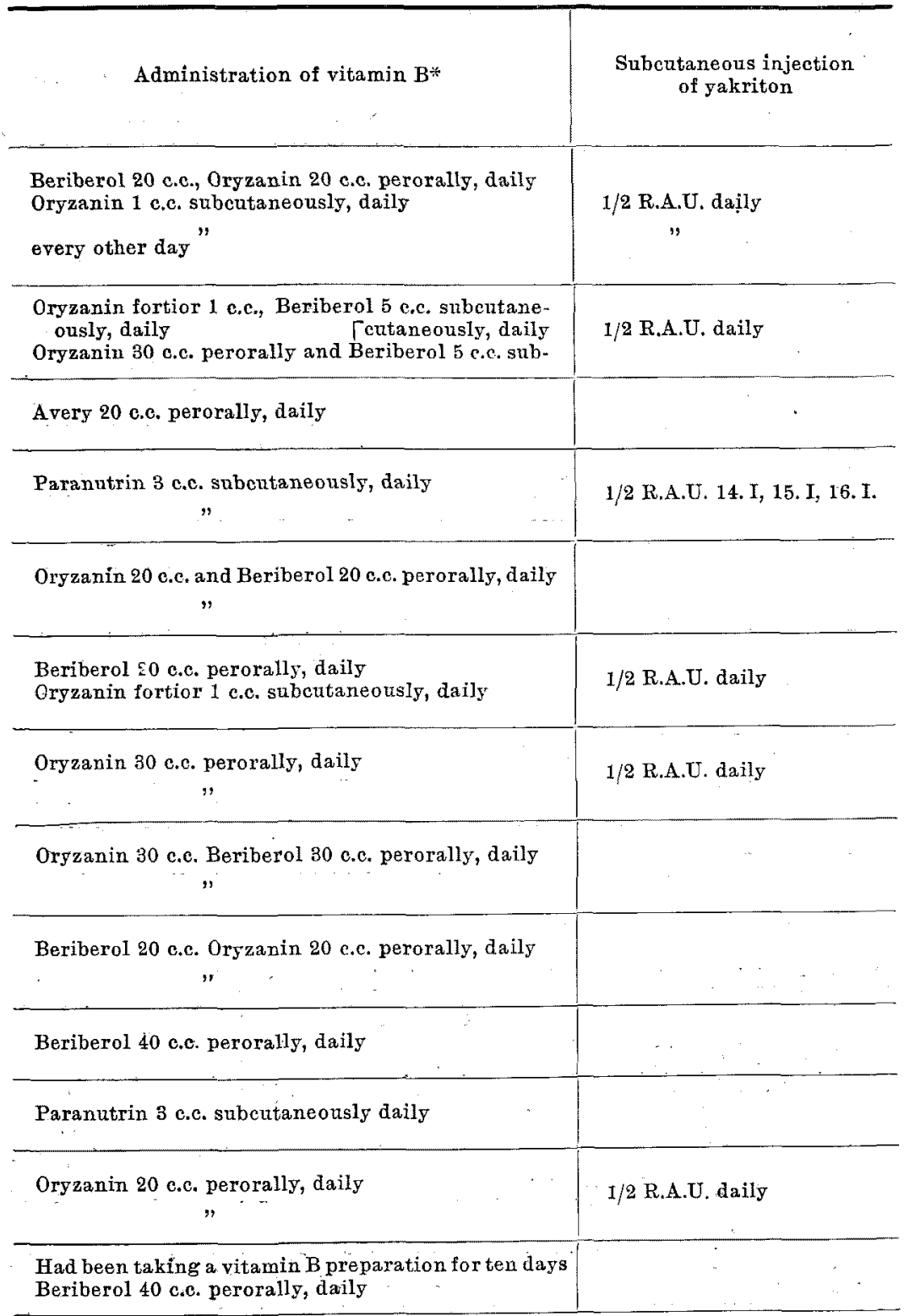


Table 2. (Continued).

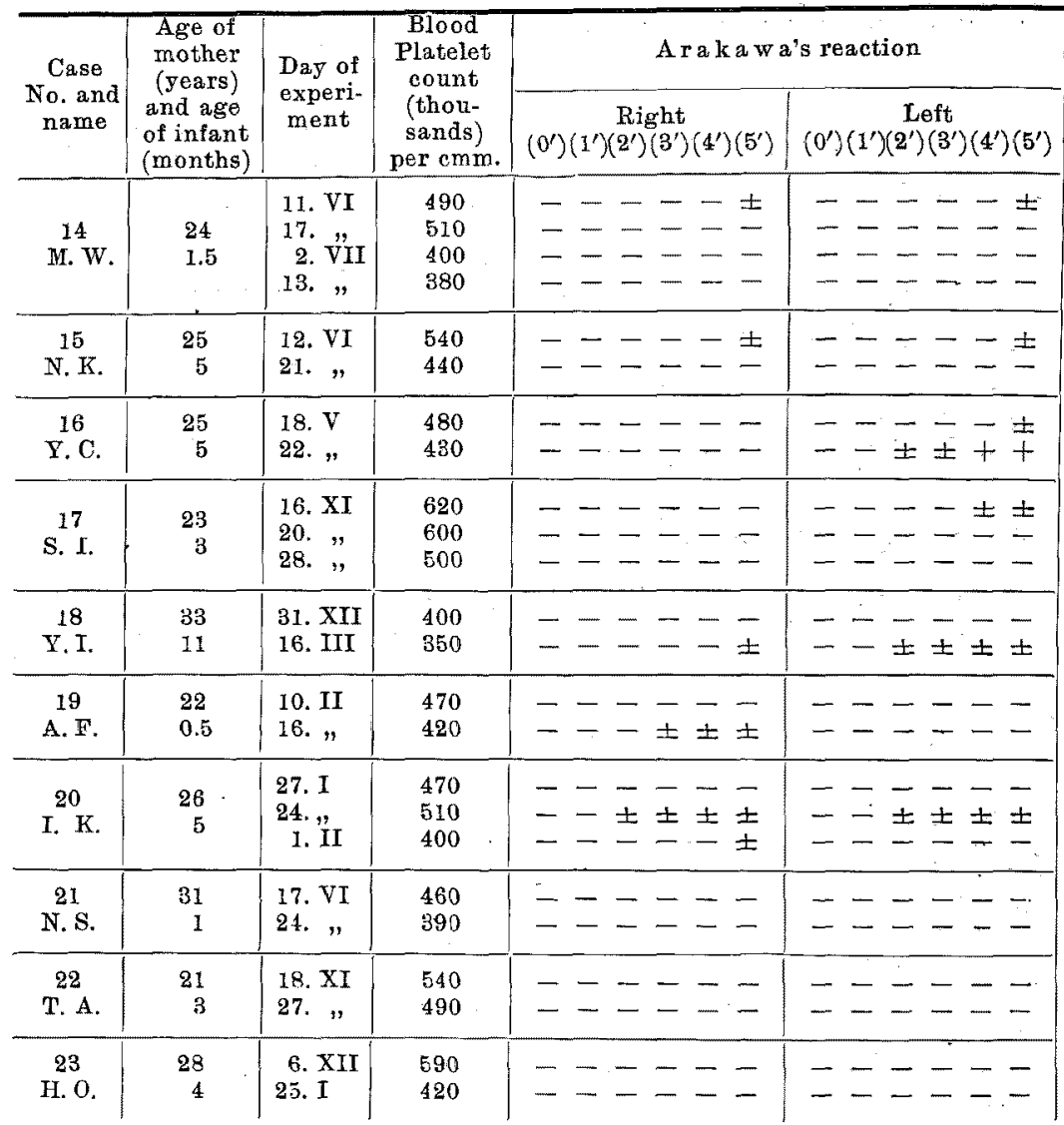

* Cf. Foot-note to Table 1.

tive to positive or to normal mostly within three weeks of the course, and the high blood platelet count decreased to a normal or physiological count (Cf. my preceding paper $\left.{ }^{11}\right)$. In Case No. 1 I found that by stopping an administration of vitamin $\mathrm{B}$ and yakriton the normal platelet count increased again to 480 thousands. In Case No. 7, the platelet count had not yet become normal, although A raka wa's reaction became normally positive. The decrease of the count however was striking.

In Cases Nos. 9, 10, 11, 12, 13, 14, 15, 16, 18, 20, 23, 25 and 26 Arakawa's reaction showed a considerable improvement, though the 


\begin{tabular}{|c|c|}
\hline Administration of vitamin $B^{*}$ & $\begin{array}{l}\text { Subcutaneous injection } \\
\text { of yakriton }\end{array}$ \\
\hline $\begin{array}{c}\text { Oryzanin } 20 \text { c.c. perorally, daily } \\
", \\
"\end{array}$ & 1/2 R.A.U. every other day \\
\hline Oryzanin 20 c.c. Beriberoal 20 perorally, daily & \\
\hline Oryzanin 30 c.c. perorally, daily & \\
\hline $\begin{array}{c}\text { Paranutrin } 5 \text { c.c. subcutaneously, daily } \\
",\end{array}$ & $\begin{array}{l}\text { 1/2 R.A.U. daily } \\
\text { " }\end{array}$ \\
\hline $\begin{array}{c}\text { Used to take Avery } \\
\qquad "\end{array}$ & \\
\hline $\begin{array}{l}\text { Had been taking oryzanin } \\
\text { Oryzanin } 30 \text { c.c. perorally, daily }\end{array}$ & \\
\hline $\begin{array}{l}\text { Had been taking a vitamin B preparation } \\
\text { Paranutrin } 5 \text { c.c. subcutaneously, daily } \\
\text { Paranutrin } 3 \text { c.c. subeutaneously, daily }\end{array}$ & 1/2 R.A.U. daily \\
\hline Beriberol 30 c.c. perorally, daily & \\
\hline Beriberol 30 c.c. perorally, daily & . \\
\hline $\begin{array}{l}\text { Oryzanin fortiro } 1 \text { c.c. } 3 \text { times and oryzanin } 30 \text { c.c. } \\
\text { perorally, daily }\end{array}$ & ' \\
\hline
\end{tabular}

normal reaction was not attained. And in Cases Nos. 9, 20, 25 and 26 the platelet count did not come down to the normal value either. In Cases Nos. 19, 21, 27, 28 and 29, though the improvement of A rakawa's reaction was not very remarkable, the platelet count decreased to the normal value.

In Cases Nos. 10, 11, 14, 19, 24, 27 and 29 mothers had been taking a vitamin $B$ preparation for some time already before the first blood examination (Cf. Table 1 ).

2. In 23 cases in Table 2 , a restoration of blood platelet count due to an administration of vitamin $B$ was indeed seen, but Araka- 
TABLE

Group of lactatiny mothers, in whom neither a restoration of blood platelet

\begin{tabular}{|c|c|c|c|c|c|}
\hline \multirow{2}{*}{$\begin{array}{c}\text { Case } \\
\text { No. and } \\
\text { name }\end{array}$} & \multirow{2}{*}{$\begin{array}{l}\text { Age of } \\
\text { mother } \\
\text { (years) } \\
\text { and age } \\
\text { of intant } \\
\text { (months) }\end{array}$} & \multirow{2}{*}{$\begin{array}{c}\text { Day of } \\
\text { experi- } \\
\text { ment }\end{array}$} & \multirow{2}{*}{$\begin{array}{l}\text { Blood } \\
\text { Platelet } \\
\text { count } \\
\text { (thou- } \\
\text { sands) } \\
\text { per cmm. }\end{array}$} & \multicolumn{2}{|c|}{ Arakawa's reaction } \\
\hline & & & & $\underset{\left(0^{\prime}\right)\left(1^{\prime}\right)\left(2^{\prime}\right)\left(3^{\prime}\right)\left(4^{\prime}\right)\left(5^{\prime}\right)}{\text { Right }}$ & $\frac{\text { Left }}{\left(0^{\prime}\right)\left(1^{\prime}\right)\left(2^{\prime}\right)\left(3^{\prime}\right)\left(4^{\prime}\right)\left(5^{\prime}\right)}$ \\
\hline $\begin{array}{c}1 \\
K . \\
K\end{array}$ & $\begin{array}{l}26 \\
18\end{array}$ & $\begin{array}{l}\text { 30. XI } \\
\text { 6. XII } \\
14 ., "\end{array}$ & $\begin{array}{l}400 \\
420 \\
390\end{array}$ & $\begin{array}{l}- \pm++ \pm+1 \\
- \pm \pm \pm+ \pm+4\end{array}$ & $\begin{array}{l}- \pm+ \pm+4 \\
- \pm+ \pm-1+\end{array}$ \\
\hline$\stackrel{2}{\text { K. }}$. & $\begin{array}{l}30 \\
6\end{array}$ & $\begin{array}{l}\text { 19. III } \\
\text { 12. IV } \\
18 .,\end{array}$ & $\begin{array}{l}410 \\
530 \\
420\end{array}$ & $\begin{array}{l}---\overline{-}- \\
--- \pm \pm \\
-----\end{array}$ & $\begin{array}{l}---\overline{-}- \\
-- \pm \pm \pm \\
-- \pm-\end{array}$ \\
\hline T. $\stackrel{3}{s .}$ & $\begin{array}{l}35 \\
15\end{array}$ & $\begin{array}{l}\text { 19. } \mathrm{X} \\
23 . "\end{array}$ & $\begin{array}{l}680 \\
760\end{array}$ & $\begin{array}{l}- \pm \pm+ \pm+ \\
-- \pm-1\end{array}$ & $\begin{array}{l}- \pm \pm \pm \pm+ \\
-- \pm-\end{array}$ \\
\hline $\begin{array}{c}4 \\
\text { H. T. }\end{array}$ & $\begin{array}{l}30 \\
10\end{array}$ & $\begin{array}{l}\text { 10. VI } \\
17 .\end{array}$ & $\begin{array}{l}440 \\
410\end{array}$ & $\begin{array}{l}-- \pm \pm \pm \pm \\
-- \pm \pm \pm\end{array}$ & $\begin{array}{l}- \pm \pm \pm \pm \pm \\
-\leftarrow \pm \pm\end{array}$ \\
\hline$\stackrel{5}{\mathrm{I}} \mathrm{Y}$. & $\begin{array}{r}24 \\
1\end{array}$ & $\begin{array}{l}\text { 13. I } \\
18 . " \\
25 . "\end{array}$ & $\begin{array}{l}540 \\
450 \\
580\end{array}$ & $\begin{array}{l}---\overline{-}- \\
--\overline{ \pm} \pm \\
-- \pm+\end{array}$ & $\begin{array}{l}------ \\
--\overline{-}-\overline{+} \\
-- \pm+1\end{array}$ \\
\hline $\begin{array}{c}6 \\
\text { T. M. }\end{array}$ & $\begin{array}{r}30 \\
5\end{array}$ & $\begin{array}{l}13 . \mathrm{I} \\
20 .,\end{array}$ & $\begin{array}{l}480 \\
450\end{array}$ & - \pm \pm \pm \pm+ & $\begin{array}{l}----\overline{-} \\
---i-\end{array}$ \\
\hline U. $\stackrel{7}{\mathrm{~K}}$ & $\begin{array}{r}2 b \\
1\end{array}$ & $\begin{array}{l}\text { 3. VI } \\
17 . " 1\end{array}$ & $\begin{array}{l}400 \\
430\end{array}$ & $\begin{array}{l}----\div \\
--\cdots--\end{array}$ & $\begin{array}{l}----- \\
--- \pm\end{array}$ \\
\hline $\begin{array}{c}8 \\
\text { A. T. }\end{array}$ & $\begin{array}{r}26 \\
3\end{array}$ & $\begin{array}{l}\text { 12. VI } \\
17 . ", \\
26 . "\end{array}$ & $\begin{array}{l}350 \\
370 \\
310\end{array}$ & $\begin{array}{l}-----\cdots \\
------\end{array}$ & $\begin{array}{l}------ \\
------ \\
----1\end{array}$ \\
\hline Y. H. & $\begin{array}{r}24 \\
9\end{array}$ & $\begin{array}{l}\text { 12. XI } \\
\text { 18. }, .\end{array}$ & $\begin{array}{l}460 \\
470\end{array}$ & $\begin{array}{l}--\cdots-\cdots \\
----\end{array}$ & $\begin{array}{l}--- \pm-- \\
--- \pm\end{array}$ \\
\hline $\begin{array}{l}10 \\
\mathrm{~K} \cdot \mathrm{X}\end{array}$ & $\begin{array}{r}27 \\
2\end{array}$ & $\begin{array}{l}\text { 16. XII } \\
2 \%, \quad,\end{array}$ & $\begin{array}{l}370 \\
360\end{array}$ & $-\cdots---\cdots$ & $\begin{array}{l}----- \\
-m--\end{array}$ \\
\hline
\end{tabular}

* Cf. Foot-note to Table 1.

w.a's reaction did not show a remarkable improvement. In Cases Nos. $1,2,4,5,6,7,12,14,17$ and 20 , yakriton was also used. In Cases Nos. $13,18,19$ and 20 , mothers had been taking vitamin $B$ before the event of my experiment. In Cases Nos. 2, 7, 9, 13, 14, 18, 20 and 21, the high platelet count decreased to the normal. In other Cases Nos. $1,3,4,6$, $8,10,11,12,15,16,17,19,22$ and 23 , the platelet count decreased too, but did not yet arrive at a normal value (Cf. Table 2 and Remarks).

3. Table 3 showed that, in 10 cases, neither a decrease of the platelet count nor an improvement of A raka wa's reaction was seen. 
3.

count nor an improvement of A rak awa's reaction was seen.

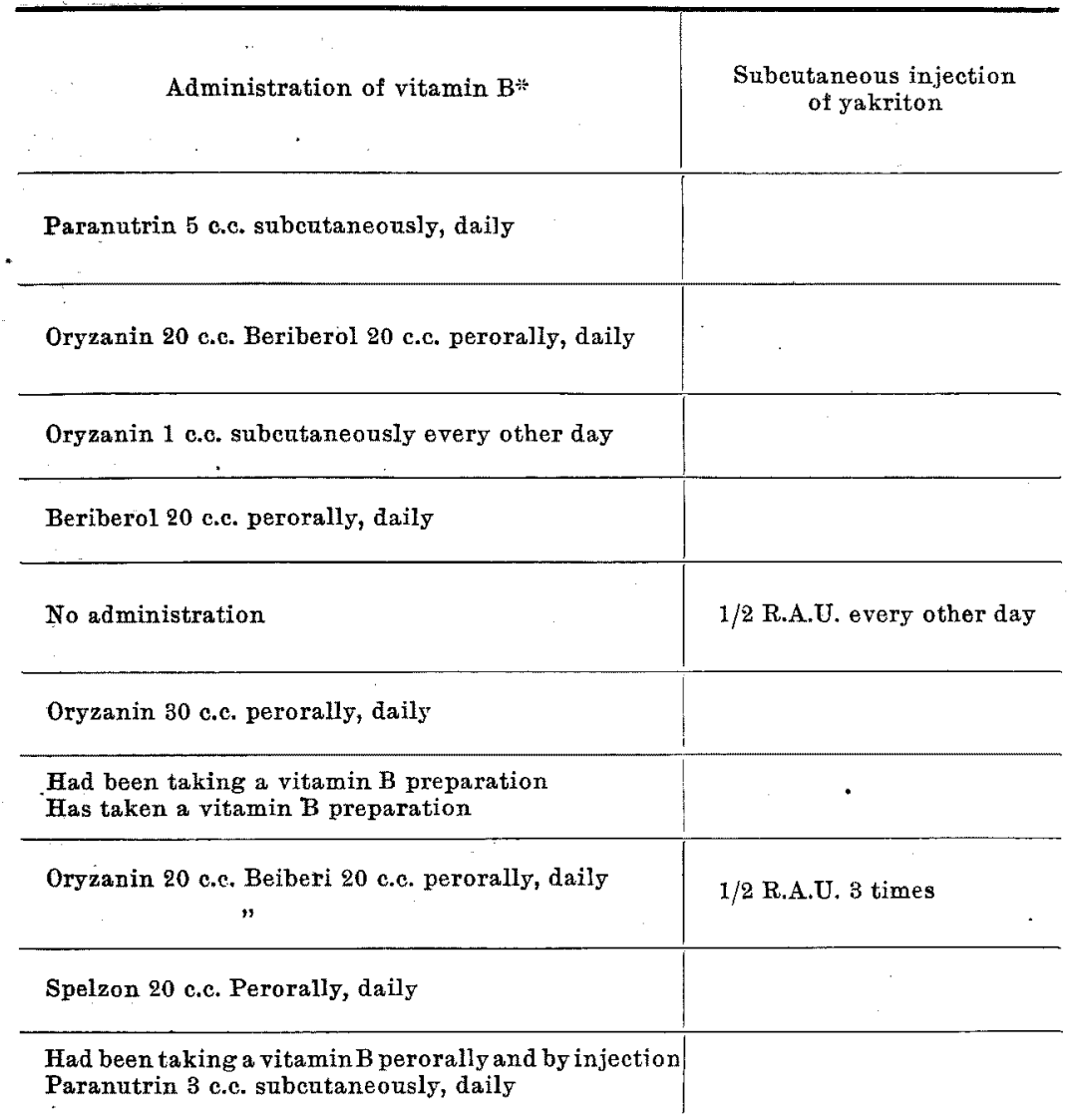

But most of them did not show a remarkable high platelet count at the first examination; the count was even normal in Cases Nos. 1, 7, 8 and 10. Only in Cases Nos. 3 and 5 , a remarkable count was seen. In Case No. 5, in whom vitamin B was not administered at all but who was injected with yakriton, a decrease of the platelet count occurred once, but the count increased again. In Cases Nos. 7 and 10 mothers who had previously been taking vitamin $B$, the platelet count was almost normal throughout the experiment (Cf. Table 3 and Remarks).

4. The relationship between blood platelet count and A raka- 
TAble 4.

Group of lactating mothers, untreated with vitamin $B$.

\begin{tabular}{|c|c|c|c|c|c|}
\hline \multirow{2}{*}{$\begin{array}{l}\text { Case } \\
\text { No. and } \\
\text { name }\end{array}$} & \multirow{2}{*}{$\begin{array}{l}\text { Age of } \\
\text { mother } \\
\text { (sears) } \\
\text { and age } \\
\text { of infant } \\
\text { (months) }\end{array}$} & \multirow{2}{*}{$\begin{array}{l}\text { Day of } \\
\text { experi- } \\
\text { ment }\end{array}$} & \multirow{2}{*}{$\begin{array}{c}\text { Blood } \\
\text { Platelet } \\
\text { count } \\
\text { (thou- } \\
\text { sands) } \\
\text { per cmm. }\end{array}$} & \multicolumn{2}{|c|}{ Arakawa's reaction } \\
\hline & & & & $\underset{\left(0^{\prime}\right)\left(1^{\prime}\right)\left(2^{\prime}\right)\left(3^{\prime}\right)\left(4^{\prime}\right)\left(5^{\prime}\right)}{\text { Right }}$ & $\frac{\text { Left }}{\left(0^{\prime}\right)\left(1^{\prime}\right)\left(2^{\prime}\right)\left(3^{\prime}\right)\left(4^{\prime}\right)\left(5^{\prime}\right)}$ \\
\hline I. $\stackrel{1}{\mathrm{~s}}$. & $\begin{array}{r}44 \\
6\end{array}$ & $\begin{array}{l}\text { 19. XII } \\
12 . \mathrm{I}\end{array}$ & $\begin{array}{l}380 \\
400\end{array}$ & $\begin{array}{l}H \text { H H H H } \\
++H \text { H H H }\end{array}$ & 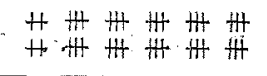 \\
\hline N. ${ }^{2}$. & $\begin{array}{l}30 \\
11\end{array}$ & $\begin{array}{l}\text { 21. XII } \\
27 . ",\end{array}$ & $\begin{array}{l}380 \\
430\end{array}$ & $\begin{array}{l} \pm \#+H+H+H \\
\pm+H H H H\end{array}$ & $\begin{array}{l} \pm+4 H \text { H H } \\
\pm+H H+H\end{array}$ \\
\hline $\begin{array}{c}3 \\
\mathrm{Y} . \mathrm{T}\end{array}$ & $\begin{array}{l}30 \\
21\end{array}$ & $\begin{array}{l}\text { 11. III } \\
16 . ",\end{array}$ & $\begin{array}{l}360 \\
360\end{array}$ & $\begin{array}{l} \pm++H+H+H \\
=+4+4+4 H\end{array}$ & $\begin{array}{l}+H+H \text { H H } \\
\pm H \text { H } H \text { H H }\end{array}$ \\
\hline M. $\stackrel{4}{\mathrm{H} .}$ & $\begin{array}{r}29 \\
7\end{array}$ & $\begin{array}{l}\text { 18. IX } \\
\text { 1. X }\end{array}$ & $\begin{array}{l}600 \\
520\end{array}$ & $\begin{array}{l} \pm \pm++++ \\
\pm \pm++++\end{array}$ & $\begin{array}{l} \pm \pm+ \pm+ \\
\pm \pm \pm++\end{array}$ \\
\hline E. ${ }^{5}$. & $\begin{array}{l}28 \\
24\end{array}$ & $\begin{array}{l}\text { 4. IV } \\
\text { 12. " } \\
20 . "\end{array}$ & $\begin{array}{l}570 \\
450 \\
390\end{array}$ & $\begin{array}{l}- \pm+++t \\
- \pm+H+H+H \\
\pm+H+H+H\end{array}$ & $\begin{array}{l}- \pm++H+H \\
- \pm+H+H \\
\pm++H+H\end{array}$ \\
\hline T. $\stackrel{6}{\mathrm{~N}}$. & $\begin{array}{l}25 \\
10\end{array}$ & $\begin{array}{l}\text { 2. } \mathrm{VI} \\
16 . n\end{array}$ & $\begin{array}{l}350 \\
360\end{array}$ & - 士 + H H H & 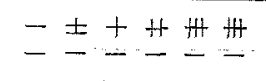 \\
\hline T. $\stackrel{7}{N}$. & $\begin{array}{l}25 \\
10\end{array}$ & $\begin{array}{l}24 . \text { II } \\
29 . ",\end{array}$ & $\begin{array}{l}590 \\
570\end{array}$ & $\begin{array}{l}-++H H+H \\
--1+\end{array}$ & - \pm \pm \pm \pm+ \\
\hline$M .8$. & $\begin{array}{l}29 \\
17\end{array}$ & $\begin{aligned} \text { 26. XI } \\
\text { 5. XII }\end{aligned}$ & $\begin{array}{l}520 \\
530\end{array}$ & $\begin{array}{l}- \pm \pm \pm \pm \pm \\
- \pm \pm \pm \pm \pm\end{array}$ & $\begin{array}{l}- \pm+4+4 \\
- \pm++4+\end{array}$ \\
\hline$\stackrel{9}{\text { M. A. }}$ & $\begin{array}{r}33 \\
4\end{array}$ & $\begin{array}{l}\text { 17. VI } \\
24 . ",\end{array}$ & $\begin{array}{l}560 \\
620\end{array}$ & $\begin{array}{l}-\overline{ \pm} \pm \pm \pm \\
\pm\end{array}$ & $\begin{array}{l}- \pm \pm++4 \\
- \pm+++4\end{array}$ \\
\hline $\begin{array}{c}10 \\
\text { J. T. }\end{array}$ & $\begin{array}{r}35 \\
4\end{array}$ & $\begin{array}{l}\text { 18. IX } \\
24 . ",\end{array}$ & $\begin{array}{l}540 \\
490\end{array}$ & $\begin{array}{l}- \pm \pm+ \pm \\
-- \pm- \pm\end{array}$ & $\begin{array}{l}-- \pm++t \\
-- \pm \pm \pm+\end{array}$ \\
\hline $\begin{array}{c}11 \\
T . K .\end{array}$ & $\begin{array}{r}23 \\
6\end{array}$ & $\begin{array}{l}\text { 14. XII } \\
19 . ",\end{array}$ & $\begin{array}{l}500 \\
530\end{array}$ & $\begin{array}{l} \pm \pm+ \pm+ \\
- \pm++4\end{array}$ & $\begin{array}{l} \pm+t+ \pm+1 \\
\therefore- \pm \pm\end{array}$ \\
\hline
\end{tabular}

wa's reaction in 11 mothers who were neither treated with vitamin $B$ nor with yakriton is shown in Table 4 . In these cases a marked change of blood platelet count and Arakawa's reaction were not seen, except for Case No. 5 (Cf. Table 4).

Of these control cases there were only 11 cases, while a larger number would have been desirable. As stated above, the present trial was a clinical experiment. And it is very difficult to leave mothers untreated for a long time, especially if their babies are very sick. A pediatrician will realize how difficult it was to collect even so small a number as 11 cases as in my own experiment. 


\section{Comment.}

An increase of blood platelet count in beriberi was reported by Ido, ${ }^{6)}$ Kubo, ${ }^{7)}$ Nakamura ${ }^{8)}$ and Iijima9). Nakamura ${ }^{8)}$ and Kō$\mathrm{da}^{12)}$ further observed a decrease of blood platelet count in cases of beriberi treated with vitamin B. A number of papers from our Laboratory stated that Arakawa-negative mothers or mothers with milk negative to A rakawa's reaction were more or less in a state of $B$ avitaminosis.

J. Kimura ${ }^{3-5)}$ and $\mathrm{I}^{1 / 2)}$ reported that a high platelet count was seen in Arakawa-negative mothers. So it is with good reason to deduct than an increase of the platelet count in Arakawa-negative mothers in due to $\mathrm{B}$-avitaminosis. In a preceding paper ${ }^{2)} \mathrm{I}$ reported that factating mothers with Arakawa-negative milk who had previously been taking vitamin $\mathrm{B}$ showed generally a lower value of the platelet count in comparison with those (with Arakawa-negative milk) who had not been taking the vitamin. This fact will also show that vitamin $\mathrm{B}$ had a platelet reducing effect in Arakawa-negative mothers.

Now, is vitamin B really effective in making the high platelet count seen in Arakawa-negative mothers decrease, if the increase of the count is due to B-avitaminosis?

52 Cases $(90 \%)$ out of $58^{*}$ mothers with a high platelet count showed a decrease of the count in the course of treatment with vitamin $\mathrm{B}$ (Cf. Tables 1, 2 and 3). . The restoration even to the normal count occurred in 33 cases $(57 \%)$. In the other 19 cases (33\%), the platelet count was not completely restored to normal. However, it was not difficult to suppose that, if the treatment with vitamin $B$ had continued longer, these 19 cases could also have shown a complete restoration of the count to the normal. In only 6 cases $(11 \%)$ out of 58 mothersNos. 2, 3, 4, 5, 6 and 9 in Table 3-an effect or vitamin B upon blood platelet count was not seen, but it is possible that the treatment with vitamin $B$ in these cases was insufficient. As regards this point see Remarks.

Arakaw a's reaction improved in 29 cases ( $47 \%$ ) out of 62 cases, and in 12 cases $(19 \%)$ out of them the complete restoration to the normal occurred. It will thus be said that, in the case of Arakawa-negative mothers treated with vitamin $B$, the restoration of the platelet

12) S. Kōda, Zika Zassi, $1935,607$.

* 4 cases (Nos. 1, 7,8 and 10) out of 62 cases, seen in Table 3 , showed a normal count of platelets at the first. 
count was more rapid than the improvement of A rakaw a's reaction. This reminds me of what Takamatsu ${ }^{13)}$ said about the relation between Arakawa's reaction and the toxicity of human milk. His opinion is : Almost all B-avitaminotic mothers seen.in daily practice are not acute cases, of course, and a daily administration of vitamin B in a not very large amount cannot easily make an Arakawa-negative case Arakawa-positive, but when mothers with Arakawa-negative milk are taking vitamin $\mathrm{B}$, the milk is losing in its toxicity or its content in glyoxal-like substance, even if it still remains'negative-to Araka wa's. reaction notwithstanding.

If I compare the above described result with that of the control experiment-11 mothers, most of them with negative Arakawa's reaction, not treated with vitamin $B$, it will be seen that the change described above was due to an administration of vitamin $B$. In these control cases a marked change of neither platelet count nor Arakawa's reaction was seen.

Thus it is indubitable that vitarnin $\mathrm{B}$ had an effect to make a high blood platelet count decrease in the case of Araka wa-negative mothers, and this is an important evidence that an abnormal increase of the platelet count in Arakawa-negative mothers is due to B-avitaminosis.

\section{Remarks.}

Such a clinical experiment as the present one would have been more remarkable if Arakawa-negative mothers with non-diseased. babies had been made objects of investigation. All the mothers in the present experiment were more or less anxious about the condition of their babies. And anxiety has a certain influence on Arakawa's reaction. We should bear this influence in mind when we consult the tables shown in the present paper.

\section{Conclusions.}

In the present paper I confirmed, in a still larger number of cases, what was previously reported by J. Kimura5). Control cases were also shown. My own conclusions are :-

1. An abnormal high blood platelet count seen in mothers secreting milk negative to A raka wa's reaction was restored to the normal value, or decreased at least on an administration of vitamin $B$.

2. A high blood platelet count is an early sign of $\mathrm{B}$-avitaminois and mothers secreting milk negative to Arakawa's reaction are in a. state of or on the brink of B-avitaminosis.

13) A. Takamatsu, Tohoku J. Exp. Med., 1934, 23, 46. 